\title{
Les bâtiments voyageurs édifiés le long de la ligne impériale
}

Buildings for travellers along the 'imperial line' (Paris-Lyons-Marseilles)

\section{François Poupardin}

\section{OpenEdition}

\section{Journals}

Édition électronique

URL : https://journals.openedition.org/rhcf/404

DOI : $10.4000 /$ rhcf.404

\section{Éditeur}

Rails \& histoire

\section{Édition imprimée}

Date de publication : 31 mai 2008

Pagination : $59-71$

ISSN : 0996-9403

Référence électronique

François Poupardin, «Les bâtiments voyageurs édifiés le long de la ligne impériale », Revue d'histoire des chemins de fer [En ligne], 38 | 2008, mis en ligne le 10 mai 2011, consulté le 22 avril 2022. URL: http://journals.openedition.org/rhcf/404 ; DOI : https://doi.org/10.4000/rhcf.404 


\title{
Les bâtiments voyageurs édifiés le long de la ligne impériale
}

\author{
François POUPARDIN
}

$\mathrm{L}$

es bâtiments des voyageurs ont sur d'autres édifices recevant du public cet avantage non négligeable d'être un extraordinaire kaléidoscope dans lequel se déroulent d'innombrables spectacles chargés d'émotions, témoin de différents modes de vie, de différentes cultures, de tragédies et de comédies qui se jouent lors des voyages. Avec les diverses constructions techniques destinées aux trains proprement dits, la gare a constitué un nouveau programme autour duquel se sont articulés de multiples réflexions qui ont généré les formes d'une architecture nouvelle ${ }^{1}$. La réalisation de «la ligne impériale », de 1849 à 1864, correspond à la formation du réseau ferré principal en France et à la fusion de certaines petites compagnies. C'est une période qui connaît de nombreux changements politiques, sociaux et économiques.

La mise en œuvre du programme du «bâtiment voyageurs » (BV) va-t-il générer des formes nouvelles et traduire sur les façades des édifices l'accueil du public et la réception des trains ? Comment la destination de l'édifice sera-t-elle représentée? L'architecture des bâtiments voyageurs de la ligne de Paris à Nice fut confiée à des maittres d'œuvre dont les séries de gares standard furent déclinées suivant l'importance du lieu à desservir. Ces gares sont l'œuvre, presque exclusivement ${ }^{2}$, de trois

1- François Poupardin, "L'architecture des bâtiments voyageurs du chemin de fer en France, des origines à la Seconde Guerre mondiale. Étude des programmes et des types », thèse de doctorat en histoire de l'art, université de Paris I - Panthéon-Sorbonne, 2005. 2- La première gare de Marseille-Saint-Charles, terminus de la ligne de Marseille à Avignon, commencée en 1847 et terminée en 1852, fut l'œuvre des ingénieurs Paulin Talabot et Henri de Dion. 
architectes, travaillant pour trois compagnies différentes, la Compagnie de Paris à Lyon, constituée en 1846, la Compagnie de Lyon à la Méditerranée, en 1852, et la Compagnie du PLM qui date de 1857.

\section{La Compagnie de Paris à Lyon}

La Compagnie de Paris à Lyon, financée par Emile Pereire et James de Rothschild, tint dès l'origine un rôle important avec une ligne de $518 \mathrm{~km}$ qui passe par Dijon et qui devait devenir l'artère prioritaire. Ses sections s'ouvrent successivement de 1849 à 1854, celle de Paris à Tonnerre, celle de Dijon à Chalon suivie par celle de Tonnerre à Dijon et, enfin, celle de Chalon à Lyon ${ }^{3}$.

\section{Les BV de I'architecte Alexis Cendrier}

Toutes les gares de Paris à Lyon sont l'œuvre de l'architecte Alexis Cendrier (1802-1893) qui fut élève de Vaudoyer et de Le Bas à l'École des Beaux-Arts, travailla pour la Compagnie de l'Ouest puis pour la Compagnie de Paris à Lyon (fig. 1 et 2).

La première gare de Lyon à Paris était à distribution latérale, avec une cour des départs et une des arrivées ${ }^{4}$. Édifiée en 1849, à simple rezde-chaussée, elle présentait des arcades régulières et, en extrémité, les pignons de ses deux halles. Celles-ci abritaient le trottoir des départs et celui des arrivées, six voies dont quatre centrales pour les manœuvres. Le bâtiment des départs, le plus important, comportait un grand vestibule, encadré de salles d'attente et d'un buffet sur un côté, d'une grande salle des bagages de l'autre. Le bâtiment des arrivées se composait principalement d'un vestibule et d'une salle des bagages ${ }^{5}$.

Les BV intermédiaires sont très nombreux entre Paris et Lyon, autour de 90. Le nombre des travées et le nombre d'étages permettent de les différencier ${ }^{6}$. À Bois-le-Roi, par exemple, le corps central de deux travées comporte un étage et des combles ${ }^{7}$. A Tonnerre, le corps

3- Les sources relatives à l'architecture de cette compagnie proviennent, entre autres, de la Revue générale de l'architecture et des travaux publics, éditée de 1840 à 1890 par l'architecte César Daly (1811-1894). Cette encyclopédie présente un intérêt particulier, reflétant les conflits de différentes doctrines relatives à l'architecture, de la tradition à la modemité. 4- Karen Bowie (dir.), Les Grandes Gares parisiennes du XIX siècle, catal. expo, Paris, Délégation à l'action artistique de la ville de Paris, s.d. [1987].

5- Cette première gare de Lyon fut remplacée par la gare actuelle, autour de 1900. 6- César Daly, «L'architecture des chemins de fer. Gare de Paris, de la ligne de Paris à Lyon, exécutée par M. A. Cendrier, architecte », Revue générale de l'architecture et des travaux publics, t. 17 et $18,1859$.

7- Ce modèle de deux travées se trouve par exemple à Maisons-Alfort, Lieusaint, Cesson, Saint-Julien-du-Sault, Briénon-sur-Armançon, Gevrey-Chambertin, Meursault ou Fontaine. 

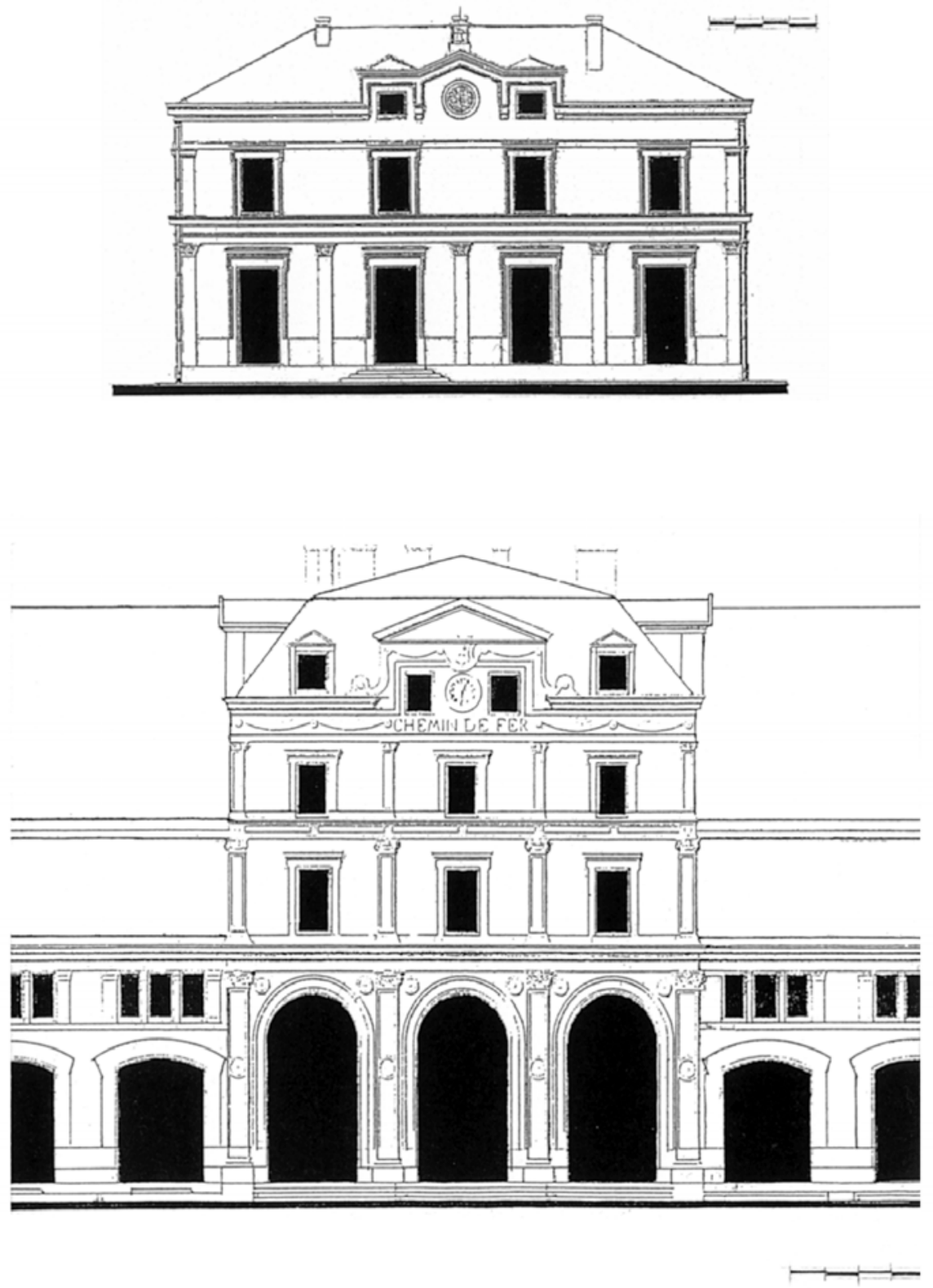

Figure 1. Les gares dessinées par Alexis Cendrier pour la Compagnie de Paris à Lyon. Façade sur cour d'une gare de première classe et de celle de Macon. Dessin François Poupardin, d'après César Daly, "L'architecture des chemins de fer. Gare de Paris, de la ligne de Paris à Lyon, exécutée par M. A. Cendrier, architecte ", Revue générale de l'architecture et des travaux publics, t. 17 et 18, 1859.

Les dessins des compagnies ont été retouchés par l'auteur de cet article dans un but de simplification afin de rendre la présentation homogène et de faire ressortir les éléments principaux des différentes morphologies. 

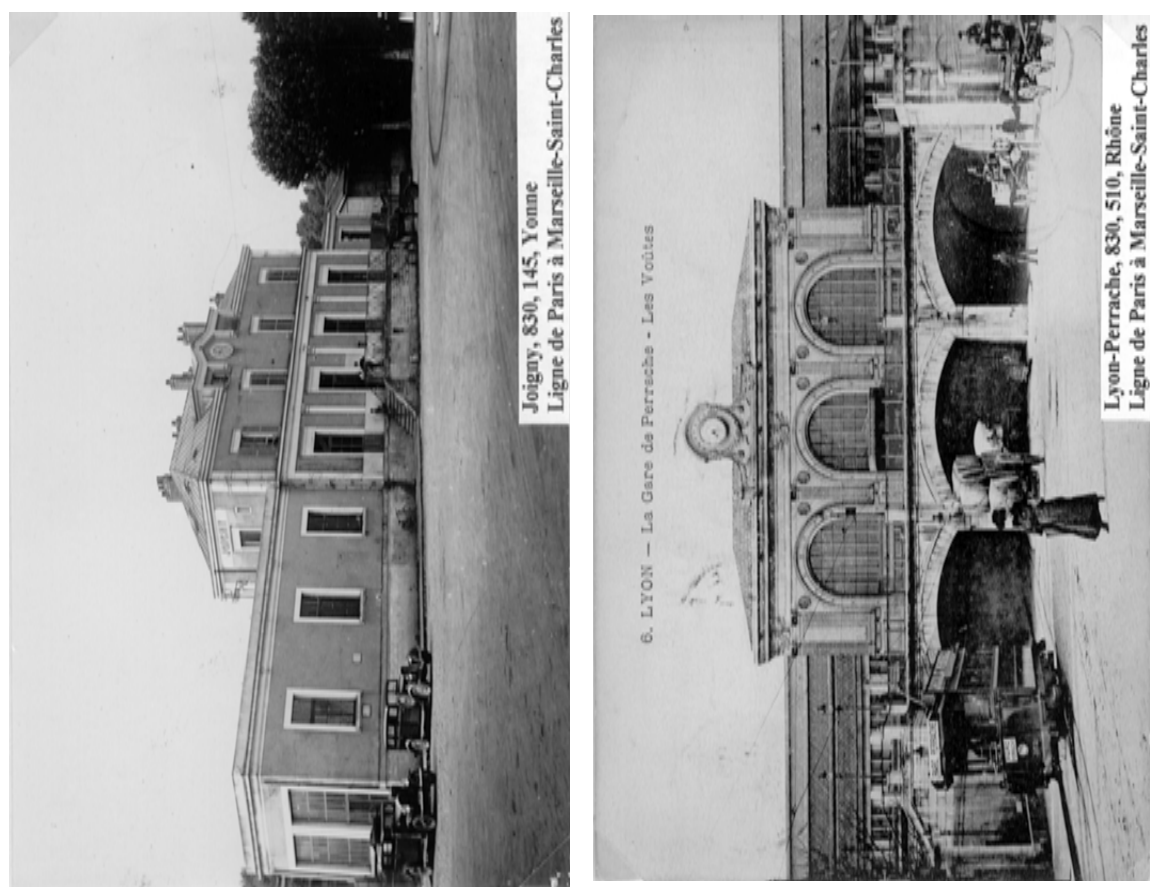

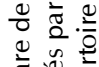

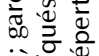

흥

$\geqslant$

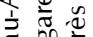

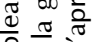

Q

竎

西

要紊

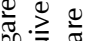

$\because$ is 0

完春要

(4)

这啳

$\pm 0$

흐웜음

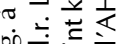

000

ดับ 잏

ठ்

$3 \frac{\pi}{4}=$

券

क० $m$
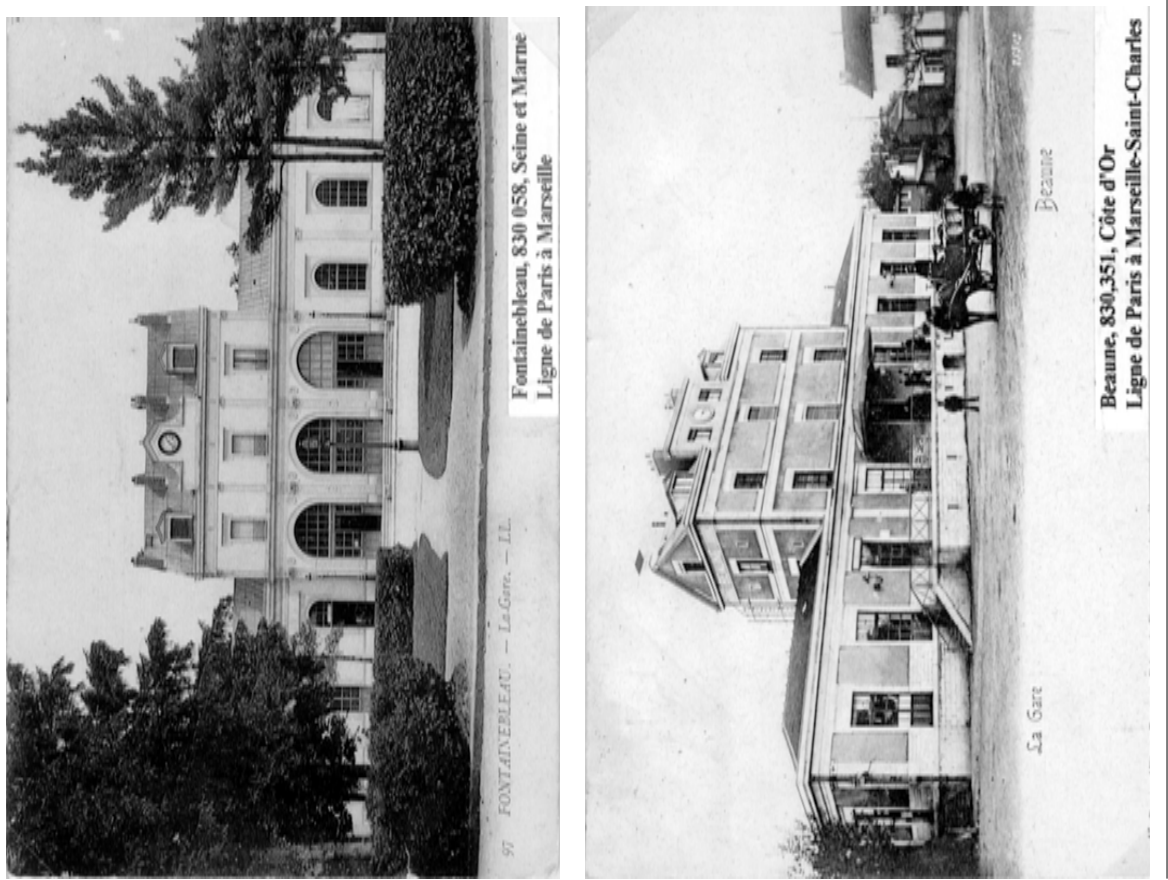

눙 $\frac{0}{2} \frac{0}{0}$

记

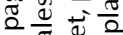

है

ن \&

존 은

ฮั

¿े

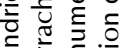

U⿺辶寸

- $\frac{\omega}{x}$ को

这交岕

원

흔

正 $m$

‥

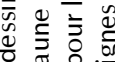

¿

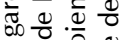

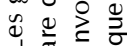

i

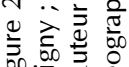

.00 .000 .0 
de bâtiment de trois travées, avec un étage et des combles, est encadré par des ailes à simple rez-de-chaussée ${ }^{8}$. A Tournus ${ }^{9}$, le corps de bâtiment, de trois travées également, comporte deux étages et des combles. Il existe deux types à quatre travées, ce sont les plus nombreux. Les bâtiments de $1^{\text {re }}$ et $2^{\text {e }}$ classe se différencient par les dimensions au sol, ainsi que par la distribution des locaux. Deux salles d'attente équipent les stations de $2^{\mathrm{e}}$ classe, alors que l'on en trouve trois dans celles de $1^{\text {re }}$ classe. Il existe néanmoins quelques gares dont les ouvertures sont plein cintre, comme à Montereau qui comporte cinq travées pour le corps principal ou à Fontainebleau dont la façade s'ouvre sur trois travées et présente un corps central en légère avancée sur les ailes. Deux gares hors classe, celles de Mâcon et de Dijon, avaient des architectures très voisines. La gare de Mâcon, édifiée en 1854, se composait d'un corps central de trois travées sur deux étages et combles ${ }^{10}$. Le vestibule s'ouvrait sur la cour par trois portes plein cintre. Les ailes à un étage comptaient neuf travées, de part et d'autre du corps central. Cette gare importante ${ }^{11}$ comportait de nombreux logements, celui du chef de gare, des sous-chefs, du receveur et de facteurs, entre autres. Une halle abritait les quais. Le bâtiment a été détruit par des bombardements de la Deuxième Guerre mondiale.

Après une gare provisoire en bois la ville de Dijon fut équipée d'un bâtiment voyageurs inauguré en même temps que la ligne de Paris à Chalon en 1851. Il se composait de deux édifices parallèles de cent vingt-cinq mètres de longueur séparés par quatre voies et trois quais. La gare va progressivement évoluer, s'agrandir de deux ailes en retour côté cour pour loger un buffet et la salle des bagages pour l'arrivée des voyageurs. Le vestibule fut également agrandi en 1865 par l'adjonction d'une avancée ouverte par trois baies plein cintre ${ }^{12}$. La gare fut dynamitée en 1944.

La gare de Lyon-Perrache fut implantée entre la Saône et le Rhône, sur la presqu'île de Perrache ${ }^{13}$. On accède à la cour des voyageurs par des rampes ou des escaliers car l'édifice est surélevé par rapport à la

8- On retrouve ce type moins répandu aux Laumes-Alésia par exemple.

9- Comme par exemple à Villefranche-sur-Saône et à Belleville-sur-Saône.

10- César Daly, art. cit.

11- Voir les illustrations et l'ouvrage de César Daly.

12- Cette gare connut ensuite de nombreux travaux de 1900 à 1909 , avec la démolition du bâtiment parallèle pour augmenter le nombre des voies, la construction d'un passage souterrain, la construction d'une halle métallique de 123,80 mètres de longueur et de 30,75 mètres de portée.

13- César Daly, art. cit. ; Pierre Chabat, Bâtiments du chemin de fer, Paris, A. Morel, 18621866. 
voirie, ce qui évite la coupure de la ville, grâce à des passages sous les voies. Le bâtiment, qui date de 1857, comporte un corps central ouvert de trois baies plein cintre et des corps en extrémité reliés par des ailes à un étage. Une halle métallique couvre six voies et trois quais.

\section{La Compagnie de Lyon à la Méditerranée}

La Compagnie de Lyon à la Méditerranée, dont Paulin Talabot fut le directeur, devient un réseau après avoir acheté d'autres lignes comme par exemple celles du Gard, du Dauphiné et de Lyon à Genève ${ }^{14}$.

\section{Les gares de Léon Grillot}

Léon Grillot (1827- ?) fut architecte du département des Vosges, architecte diocésain de Saint-Dié. Il travailla pour la Compagnie de Paris à Strasbourg, fut l'auteur des deux premières gares de Metz, une première, provisoire, en bois, puis une gare terminus à fonction latérale. Il édifia les gares des embranchements de la ligne de Paris à Strasbourg comme celui de Frouard à Forbach. Il travailla par la suite pour la Compagnie de Lyon à la Méditerranée en 1854-1855, édifia les gares entre Lyon et Avignon et reprit des éléments d'architecture de ses travaux précédents (fig. 3 et 4). Par exemple, à Dieulouard ${ }^{15}$ ou à Ars-sur-Moselle, on remarque des particularités au rez-de-chaussée, comme les baies dont l'encadrement de la partie plein cintre est en légère saillie, une résille décorative sous la toiture et un auvent bien particulier du côté du quai à Dieulouard. Par ailleurs, aussi bien la gare de Faulquemont d'origine que ses transformations montrent bien que nous avons affaire à des édifices tramés, que l'on allonge suivant l'évolution des besoins. La parenté avec la première gare d'Avignon est évidente ${ }^{16}$. Le premier train joignant Paris à Marseille s'arrête à Avignon en 1854, devant la gare de Grillot à

14- C'est Pierre Chabat, regroupant des dessins de gares dans son ouvrage cité, qui constitue nos sources sur l'architecture de ces BV. L'auteur de ce livre travaille pour les chemins de fer italiens, la Compagnie des chemins de fer du Nord et pour la Compagnie des chemins de fer du Midi. On le retrouve également impliqué dans la construction du siège du PO, 8, rue de Londres, à Paris.

15- Voir François Poupardin, «Typologie des gares rurales et de moyenne importance du Nord-Est de la France », in «Le patrimoine ferroviaire : enjeux, bilans et perspectives », actes du 6 colloque de l'AHICF, Rerne d'histoire des chemins de fer, $\mathrm{n}^{\circ}$ 20-21 (1999), p. 121. 16- À Avignon l'emplacement des voies et de la gare posait le problème de la desserte d'une ville historique encerclée de remparts. Prosper Mérimée (1803-1870), inspecteur des Monuments historiques, assisté du jeune Viollet-le-Duc, imposa un tracé des voies ferrées à l'extérieur de l'enceinte. Une porte fut créée ainsi que la rue de la République qui se dirige vers le Palais des Papes. Voir aussi Michèle Lambert, «Les voies ferrées et les gares dans les villes », $1^{\text {te }}$ partie, « Avignon », Paris, IPRAUS/AHICF, sept. 1992, 123 p. ; $2^{e}$ partie, " Nîmes, du canal au chemin de fer », ibid., 1994, 124 p. 

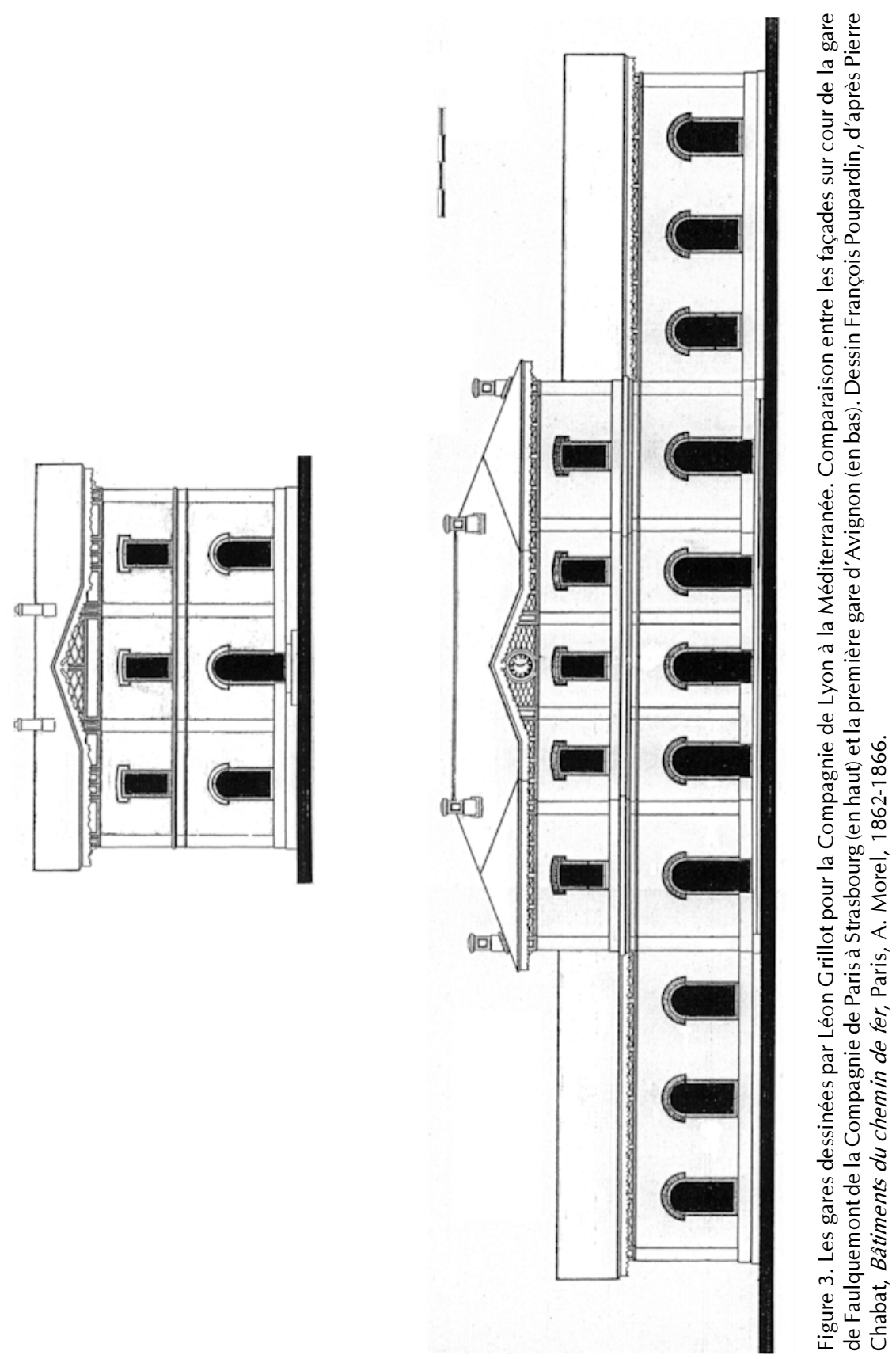

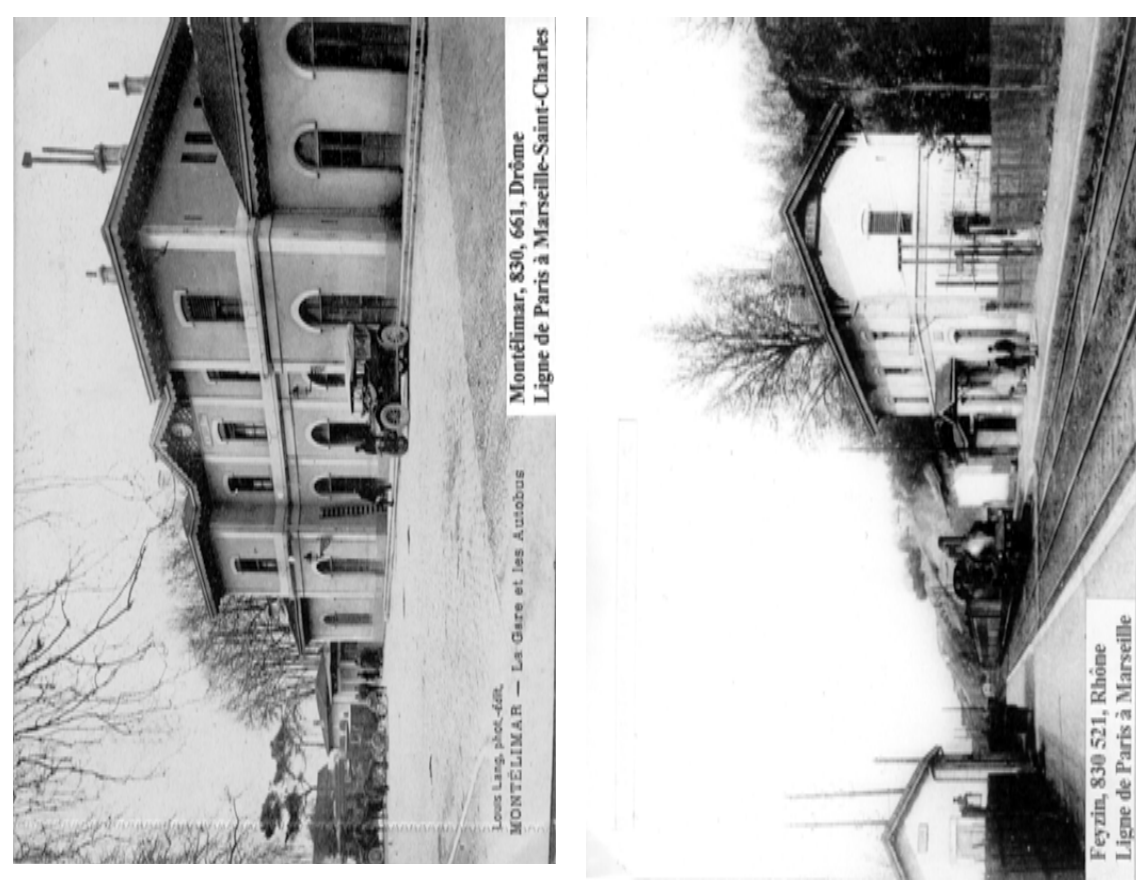

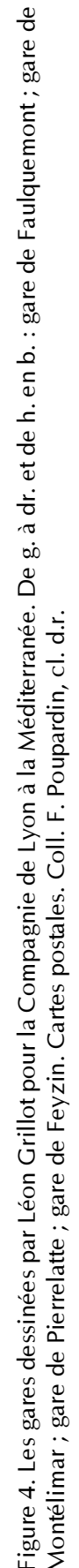
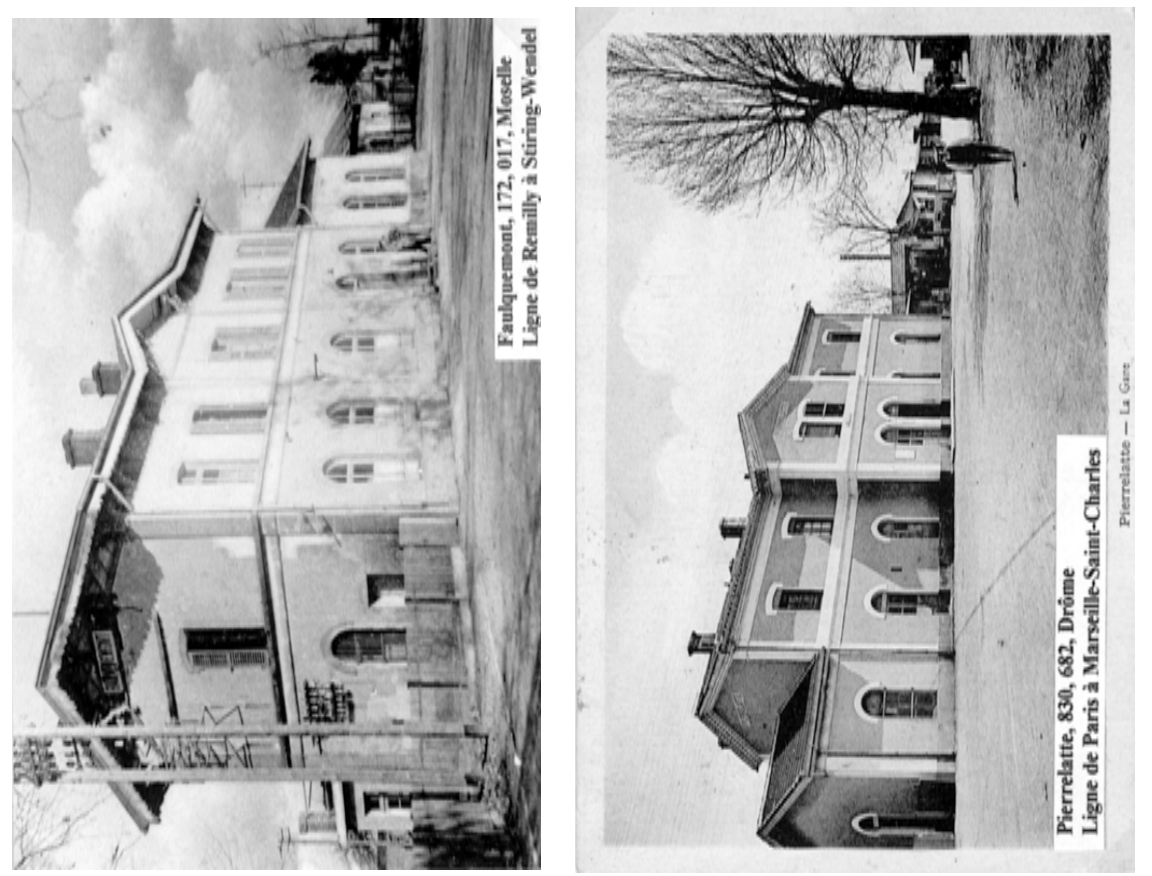
l'emplacement de la gare actuelle. Cette première gare présente un corps central à un étage de cinq travées, celles qui sont en extrémité côté cour viennent en avancée. Ce corps central dont les baies à rez-de-chaussée sont plein cintre est encadré de deux ailes basses. Une résille décorative en bois découpé orne les pignons et les rives, caractérisant comme nous l'avons déjà noté les façades étudiées par cet architecte. Ce modèle se retrouve d'ailleurs à la gare de Tain-l'Hermitage, à celles de Vienne, d'Orange et de Montélimar. À Vienne, une variante présente un corps central de cinq travées qui ne comporte pas de décrochement en façade du côté de la cour. À Pierrelatte, la gare à rez-de-chaussée et étage comporte un corps central percé d'ouvertures doubles, en avancée sur des ailes de deux travées. Les baies du rez-de-chaussée sont en plein cintre, alors que celles de l'étage sont simplement cintrées. Ce modèle se retrouve par exemple à la gare de Loriol ou de Sorgues. En revanche, à Feysin et à Saint-Fons, les gares se composent d'un corps de bâtiment de trois travées à un étage. Nous retrouvons la signature de l'architecte Léon Grillot avec un petit auvent du côté des quais.

\section{La Compagnie du PLM}

Les fusions des compagnies correspondent aux mouvements de plus grande ampleur issus des intérêts financiers qui s'expriment par les regroupements de diverses sociétés pendant le Second Empire. La création du PLM en 1857 fut un symbole du capitalisme libéral. C'est autour de l'axe de Paris à Marseille que se constitue le premier réseau français, grâce aux fusions et concessions multiples qui s'opèrent pendant cette période ${ }^{17}$.

\section{Les gares de I'architecte Jules Bouchot}

Jules Bouchot (1817-1907) fut attaché au ministère des BeauxArts, construisit le palais de justice de Tarbes et la façade du ministère de la Guerre sur le boulevard Saint-Germain à Paris. Il fut architecte en chef des bâtiments du ministère de l'Instruction publique. Il travailla pour plusieurs compagnies de chemin de fer, d'abord à Lille, puis à Milan et enfin pour le PLM (fig. 5 et 6 ).

Deux gares sont d'aspect semblable, celle de Valence édifiée en 1865 et celle d'Avignon en 1866. Les corps centraux en pierre de taille, de cinq travées, révèlent les mêmes références au classicisme. On remarque la différence de la position de l'horloge sur la façade, côté de la

17- Voir, outre de nombreux articles publiés dans La Vie du rail, Jean Chaintreau, Jacques Cuynet et Georges Mathieu, Les Chemins de fer PLM, Chanac/Paris, La Régordane/La Vie du Rail, 1993. 
cour des voyageurs. Elle est inscrite dans l'ouverture centrale à Valence, alors qu'elle domine au niveau de la balustrade à Avignon. Ici la composition de la façade du corps central est butée en extrémité par des pilastres doubles alors qu'à Valence les pilastres sont simples. La comparaison avec la façade du Petit Trianon, édifié par Gabriel en 1768, s'impose. La proportion des façades de ces deux gares s'inscrit dans un double carré, rectangle d'environ $24 \mathrm{~m}$ de large sur 12 de haut. Ces BV sont encadrés d'ailes à un étage, symétriques à Avignon, dissymétriques à Valence. Une élégante halle métallique cintrée abrite les quais en reliant deux édifices de part et d'autre des voies à Avignon. À Valence, la charpente de la halle est de forme triangulaire.

La première gare de Marseille-Saint-Charles, terminus de la ligne de Marseille à Avignon, ne fut achevée qu'en 1852, cinq ans après l'inauguration de la ligne. La gare d'origine des ingénieurs Paulin Talabot et Henri de Dion présentait en façade deux halles métalliques plein cintre, bloquées de part et d'autre par deux corps de bâtiment en avancée. La gare est à distribution latérale, avec les services consacrés au « départ » d'un côté, curieusement disposés sur la droite du BV, et le service dédié à «l'arrivée » de l'autre côté des voies. Quant au bâtiment de la cour départ, qui est à simple rez-de-chaussée, il fut agrandi par l'architecte Bouchot, en 1863, pour abriter un buffet et des bureaux du trafic ${ }^{18}$.

À Toulon, le principe de la desserte de la ville fut adopté en 1853, avec une gare implantée dans le périmètre des fortifications. La construction de la ligne fut confiée en 1856 à la Compagnie de Lyon à la Méditerranée, puis en 1857 à la nouvelle Compagnie PLM. Avec la campagne d'Italie, Toulon, arsenal militaire, recevait les troupes impériales qui étaient envoyées pour soutenir Cavour via Gênes et l'aider à repousser les Autrichiens de Lombardie. La ligne de Marseille à Toulon fut entreprise malgré les difficultés rencontrées lors de la construction de nombreux tunnels, ouvrages d'art et tranchées. À Toulon, après un premier édifice conçu par l'architecte Laroze ${ }^{19}$, la gare fut détruite par un incendie en 1868 et reconstruite avec un corps central, flanqué de deux ailes basses se retournant sur la cour des voyageurs. Le corps central comporte trois baies plein cintre et le hall se développe sur toute la hauteur de l'édifice, ce qui lui donne un caractère monumental. La partie supérieure est bordée par une balustrade et en son centre prend place une horloge. La halle qui couvre trois voies fut photographiée

18- Cette première gare de Marseille-Saint-Charles sera reconstruite en 1892 par l'architecte Antoine Bouvard. C'est la gare actuelle.

19- D’après «Les amis du vieux Toulon », article publié dans Le Toulonnais de février 1870. 

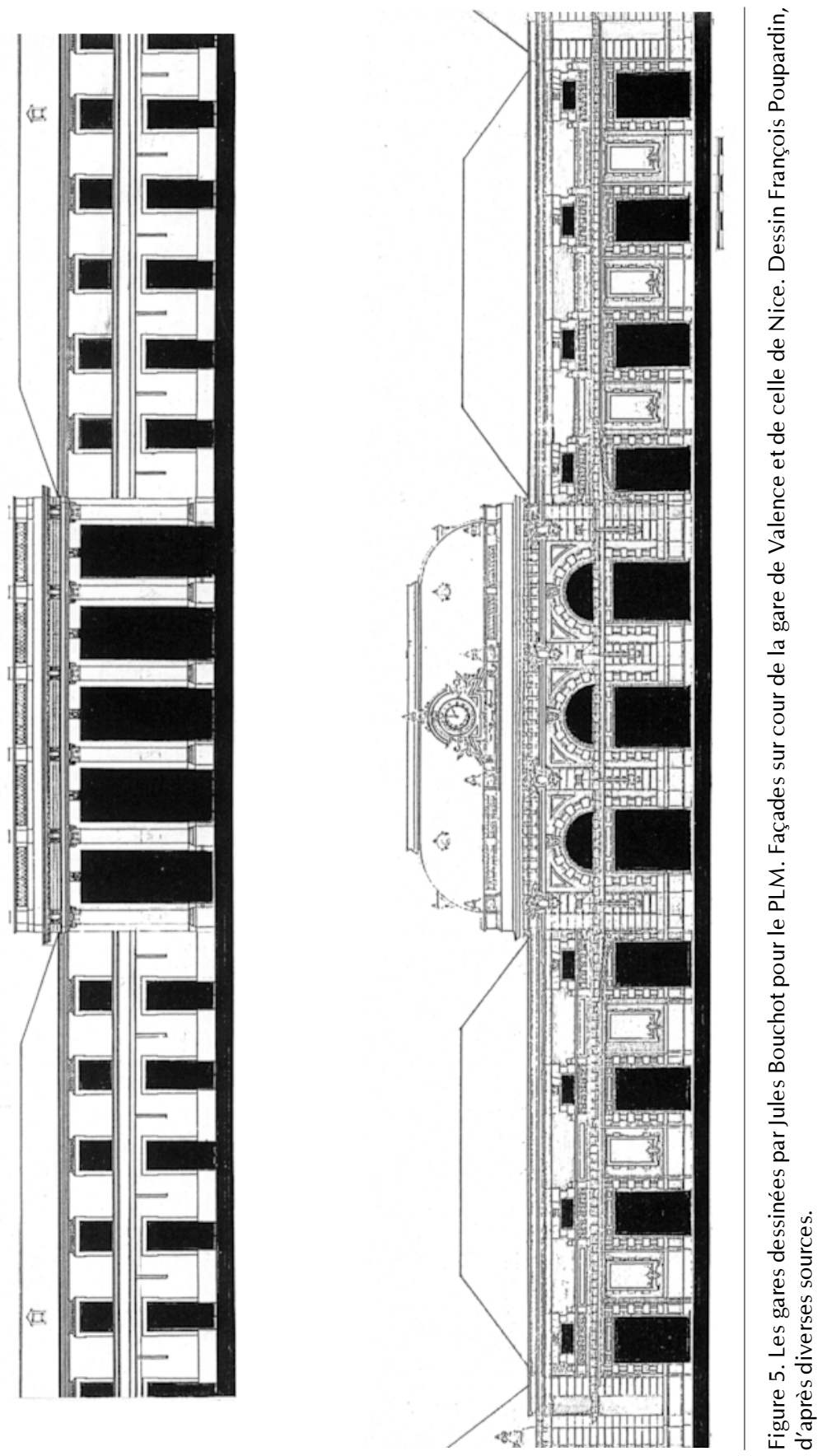

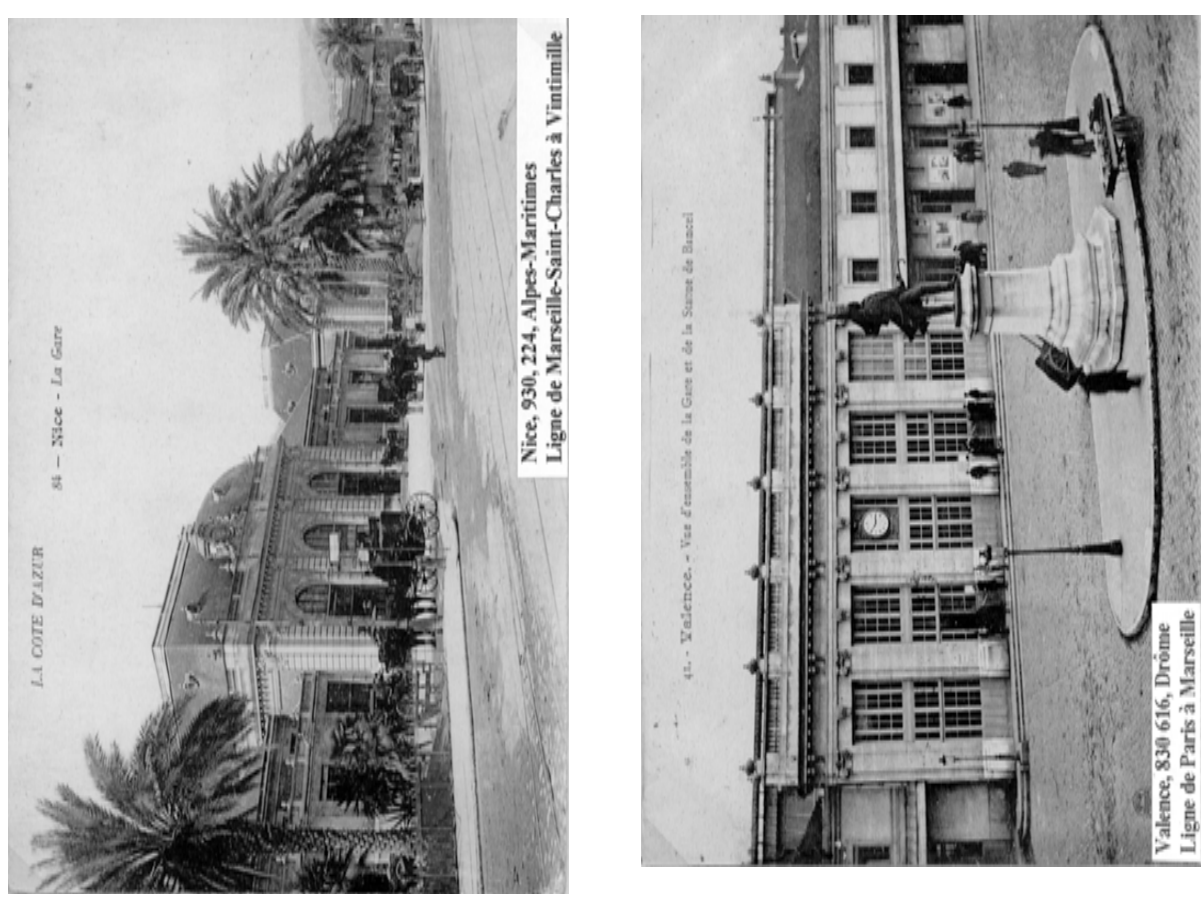

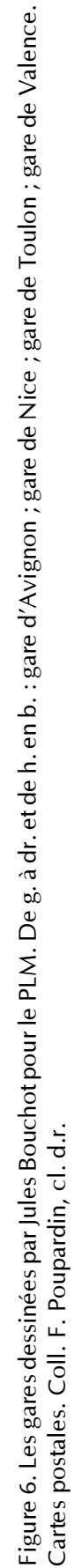
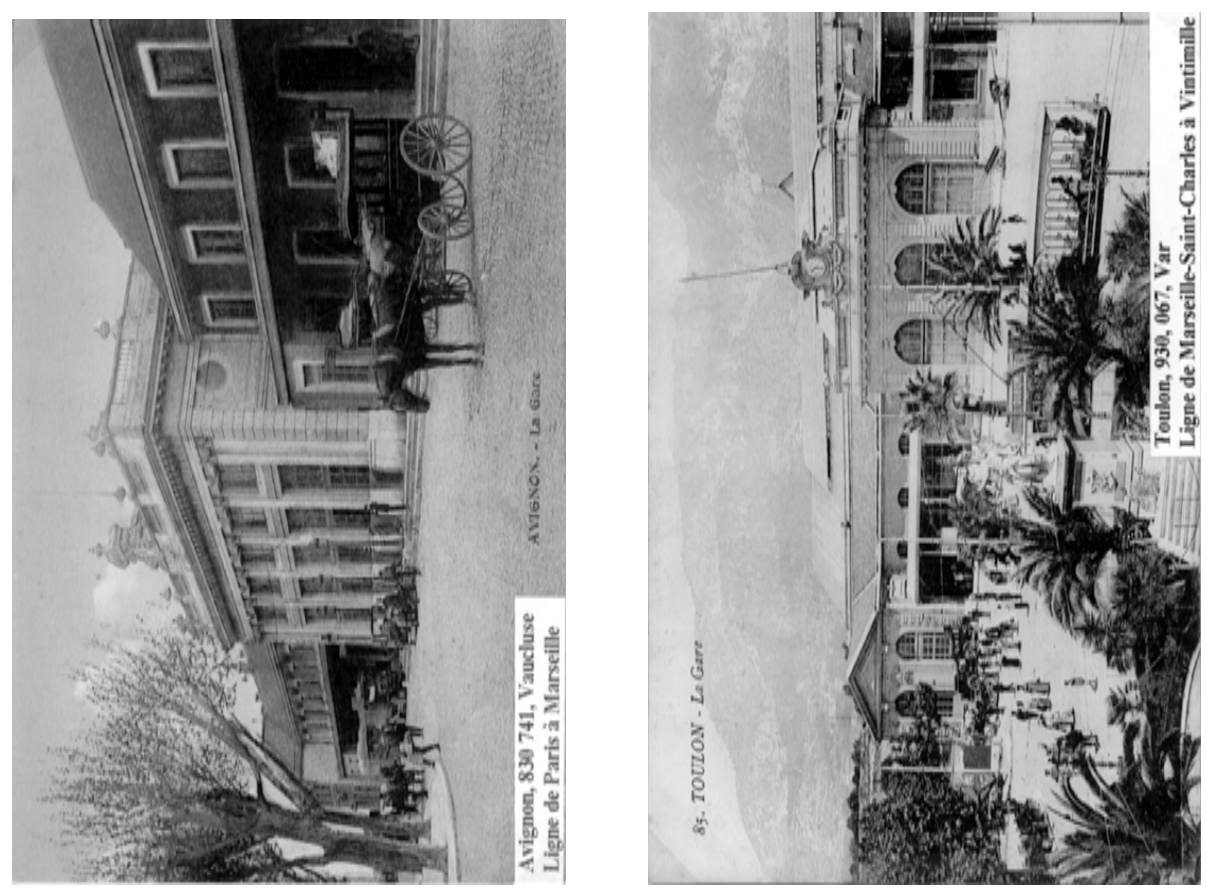
par Edouard Baldus vers 1850. Quant au bâtiment situé de l'autre côté des voies, il sera détruit pour permettre l'implantation de voies supplémentaires.

En 1860, Le comté de Nice est rattaché à la France après référendum ${ }^{20}$. À Nice le BV, qui date de 1865, comportait un bâtiment annexe de l'autre côté des voies ${ }^{21}$. Le vestibule, qui occupait toute la hauteur du corps central, était magnifiquement aménagé, comme en témoignent des gravures et des photos anciennes. La marquise de la cour des voyageurs a été ajoutée ultérieurement. Le bâtiment de service de l'autre côté des voies sera démoli et la merveilleuse halle en anse de panier qui couvre les quais doublée en 1947 pour abriter des voies supplémentaires.

Ainsi s'achève un voyage de plus de $1000 \mathrm{~km}$ de Paris à Nice. Les gares, enjeu important de la concurrence, présentent des architectures « importées » par les maitres d'œuvre retenus par les compagnies. L'intérêt des recherches des architectes, leurs constructions par lignes et par compagnies, la variété de leurs bâtiments voyageurs méritaient d'être montrés : ils font partie de notre patrimoine industriel du chemin de fer et doivent être autant mis en valeur dans leurs mutations continuelles que protégés comme témoins de l'histoire de notre architecture ${ }^{22}$.

20- De 1857 à 1864 la gare de Milan est construite par l'architecte conseil du PLM Jules Bouchot (sans doute aussi pour remercier l'Empereur de son aide militaire qui avait cinq ans plus tôt contribué au départ des Autrichiens de la capitale de la Lombardie). L'architecture de la gare de Milan est proche de celle de la gare de Nice mais en plus grand, avec cinq travées plein cintre au lieu de trois à Nice; même forme de toit, des balustrades, une horloge au centre ; les baies sont cependant séparées par des pilastres doubles. Voir Gianfranco Angeleri et Cesare Columba, Milano Centrale, storia di una starione, Rome, éd. Abete, 1985.

21- Le développement du trafic oblige à le démolir pour faire passer des voies supplémentaires en 1926.

22- Voir l'article « Rénovation des gares » dans Monuments historiques $\mathbf{n}^{\circ} 6$ (1978), dans lequel le département des Bâtiments de la SNCF, dont les dirigeants d'alors formaient une sorte d'oligarchie qui laissait peu de place à la concertation, présente la rénovation de la gare de Nice, avec des photos avant et après travaux. Le hall qui se déployait sur toute la hauteur du corps central a été tronqué pour créer des bureaux en partie supérieure! Ce même article mentionne d'autres rénovations de cette époque également très contestables comme la gare de Vichy ou celle de Metz dont les halles sur quais ont été démolies pour faire place à un parking. Un projet de parking analogue à la place de la halle couvrant les quais de la gare d'Avignon a été étudié et heureusement abandonné. Ce ne sont plus les années glorieuses de l'architecture ferroviaire, les projets étudiés par des cabinets extérieurs restent également décevants. La façade de l'hôtel de la gare de Lyon à Paris, au pied de la tour équipée d'horloges ou les « aménagements » de la gare de Lyon-Perrache en sont des exemples sur la ligne de Paris à Nice. 